\title{
Sospecha clínica, dímero D y angiotomografía de tórax para el diagnóstico de tromboembolismo pulmonar
}

Writing Group for the Christopher Study Investigators. JAMA, 2006; 295: 172-179.

\section{Objetivo}

Evaluar la efectividad clínica de un algoritmo diagnóstico simplificado basado en la sospecha clínica, el dímero D y la Angiotomografía de tórax (AngioTC) en pacientes con sospecha de tromboembolismo pulmonar (TEP).

\section{Diseño}

Estudio de cohorte, prospectivo, con un seguimiento de tres meses.

\section{Lugar \\ 12 centros de Holanda}

\section{Pacientes}

3.306 pacientes consecutivos con sospecha de TEP. Se sospechó TEP ante la aparición súbita de disnea, el empeoramiento súbito de la disnea preexistente o dolor pleurítico agudo.

\section{Intervención}

Los pacientes con sospecha de TEP fueron clasificados en dos grupos: "TEP improbable" y "TEP probable" mediante una versión dicotomizada de la probabilidad clínica de Wells para TEP, Ver tabla 1.

Tabla 1: versión dicotomizada de la regla de probabilidad clínica de Wells.

\begin{tabular}{l|c}
\multicolumn{1}{c|}{ Variable } & Puntos \\
$\begin{array}{l}\text { Semiología de trombosis venosa profunda (TVP): edema de la extremidad y dolor a la } \\
\text { palpación venosa. }\end{array}$ & 3,0 \\
\hline Diagnóstico atternativo menos probable que TEP & 3,0 \\
\hline Frecuencia cardíaca mayor de 100 latidos por minuto & 1,5 \\
\hline Inmovilización mayor a tres días o cirugía en las cuatro semanas previas & 1,5 \\
\hline Antecedente de TEP o TVP & 1,5 \\
\hline Hemóptisis & 1,0 \\
\hline Neoplasia en tratamiento actual (incluido el paliativo) o en los seis meses previos. & 1,0 \\
\hline
\end{tabular}

Probabilidad clínica de TEP improbable si 4 o menos puntos, TEP probable si alcanza más de cuatro puntos.

En los pacientes con "TEP improbable" y dímero-D negativo se consideró descartado el TEP y se suspendió la anticoagulación. Al resto de los pacientes se les realizó AngioTC (aquellos clasificados como "TEP improbable" con dímero-D positivo ó como "TEP probable"). En caso de que la AngioTC fuese normal se les suspendió la anticoagulación, considerándose descartado el TEP. A todos se los siguió durante tres meses.

\section{Medición de resultados principales}

Incidencia de eventos de tromboembolismo venoso sintomático durante los tres meses de seguimiento, definidos como tromboembolismo fatal y no fatal.

\section{Resultados principales}

De los 3306 pacientes, 1100 fueron clasificados como "TEP probable" y 2206 como "TEP improbable". Se confirmó el TEP en 37,1\% $(n=408)$ vs. $12 \% \quad(n=266)$ en cada grupo respectivamente $(\mathrm{p}<0,001)$.

Del grupo "TEP improbable", 1057ptes (32\%) tuvieron dímero-D negativo por lo que se consideró excluido el TEP y se suspendió la anticoagulación. En el seguimiento a tres meses presentaron cinco eventos tromboembólicos no fatales (0,5\% IC95\% 0,2\%-1,1\%).

Se les indicó AngioTC a 2249 pacientes (1100 con "TEP probable" y 1149 con "TEP improbable" y dímero-D positivo). 50 no pudieron hacerla por problemas técnicos y en 1505 pacientes (45\%) se excluyó TEP. Hubo 18 episodios de tromboembolismo $(1,3 \%$ IC95\% 0,7\%-2,0\%) en los tres meses de seguimiento (0,5\% IC95\% $0,2 \%-1,0 \%)$.

En 20 pacientes con AngioTC no concluyente se diagnosticaron dos TEP por centellograma de ventilación/perfusión ( $\mathrm{V} / \mathrm{Q})$ y un evento tromboembólico no fatal en el seguimiento. De los 50 pacientes a quienes no se les pudo realizar AngioTC, tres presentaron TEP por centellograma V/Q y dos, TVP.

Por último, 674 pacientes tuvieron TEP confirmado por AngioTC, 20 pacientes $(3 \%)$ evolucionaron con tromboembolismo recurrente a pesar de la anticoagulación, 11 desarrollaron TEP fatales, tres no fatales y seis, TVP. Un paciente se perdió de seguimiento y la mortalidad global fue del 7,2\%.

\section{Conclusiones}

Esta gran cohorte de 3306 pacientes consecutivos con sospecha de TEP demuestra que el uso de un algoritmo dicotómico sencillo basado en la probabilidad clínica, el dímero-D y la AngioTC puede guiar la decisión de anticoagular con un bajo riesgo de eventos tromboembólicos en el seguimiento a tres meses.

\section{Comentario}

Este algoritmo es capaz de excluir el TEP con un buen margen de seguridad en $50 \%$ de los pacientes con sospecha clínica del mismo. Un tercio de los pacientes clasificados como "TEP improbable" no necesitaron otro método diagnóstico más que el dímero$\mathrm{D}$, y solo presentaron $0,5 \%$ de incidencia de eventos tromboembóli$\cos$ no fatales en el seguimiento a tres meses.

En el resto de los pacientes, una AngioTC negativa descartó TEP sin necesidad de otro método de imágenes y la incidencia de TEP en los tres meses de seguimiento fue de 1,3, comparable a la de otros trabajos'en los que el TEP se había descartado por angiografía (1,7\%; IC95\% 1,0-2,7).

El algoritmo fue pragmático y se completó en el $98,5 \%$ de los pacientes, permitiendo tomar decisiones terapéuticas en el 97,9\% de los mismos, lo que lo hace más aplicable y sencillo que otro recientemente publicado? ${ }^{2}$.

\section{Conclusión del comentador}

Consideramos útil la validación prospectiva de un algoritmo basado en la regla de predicción clínica de Wells ${ }^{3}$ de dos alternativas de probabilidad clínica de TEP (probable ó improbable) en lugar de la que clásica que incluye tres categorías (riesgo bajo, moderado y alto) lo que facilita el manejo clínico.

Diego Goffredo [ Médico Especialista en Neumonología, Servicio de Neumonología, Hospital de Clínicas "Jose de San Martín". ]

Goffredo D. Sospecha clínica, dímero D y angiotomografía de tórax para el diagnóstico de tromboembolismo pulmonar. Evid. actual. práct. ambul. 2006; 9(3) ;79. May-Jun. 2006. Comentado de: Writing Group for the Christopher Study Investigators. JAMA 2006 Jan 11; 295: 172-179. Effectiveness of managing suspected pulmonary embolism using an algorithm combining clinical probability, D-Dimer testing, and computed tomography. PMID: 16403929.

\section{Referencias}

1. Van Beek, E.J.R., et al., Clinical Validity of a Normal Pulmonary Angiogram in Patients with Suspected Pulmonary Embolism-A Critical Review. Clinical Radiology, 2001. 56(10): p. 838-842.

2. Perrier, A., et al., Multidetector-Row Computed Tomography in Suspected Pulmonary Embolism. N Engl J Med, 2005. 352(17): p. 1760-1768.

3. Wells, P.S. and A. DR, Derivation of a Simple Clinical Model to Categorize Patients Probability of Pulmonary Embolism:Increasing the Models Utility with the SimpliRED D-dimer. Thromb Haemost, 2000. 83: p. 416-20. 